\section{Simultaneous Spin and Structure Maps by Spin-Polarized STM}

One of the current challenges for materials science is to create materials that effectively utilize electron spin. An important characterization step for such materials would be to correlate the electron spin and physical structure. In a recent report, Arthur R. Smith and co-workers from Ohio University, Athens, and Case Western Reserve University, Cleveland, obtained both the chemical and spin structures of a magnetic material from a single measurement. The researchers used spinpolarized scanning tunneling microscopy (SP-STM) to map out the atom locations and spin orientations on the (010) face of $\mathrm{Mn}_{3} \mathrm{~N}_{2}$ and compared their results to models based on first-principles calculations. According to Smith, "the development of this technique brings us one step closer to being able to utilize electron spin in science and technology."

As described in the November 25 issue of Physical Review Letters, the researchers first grew atomically smooth layers of $\mathrm{Mn}_{3} \mathrm{~N}_{2}$ on a $\mathrm{MgO}$ substrate by molecularbeam epitaxy (MBE). In situ STM characterization of the surface was carried out in an ultrahigh vacuum chamber attached to the MBE chamber. STM images were obtained at $300 \mathrm{~K}$ in the constant-current mode using both nonmagnetic (W) and magnetic (W coated with Mn or Fe) STM tips. Use of very sharp tips allowed the resolution of individual $\mathrm{Mn}$ atoms on the surface. To ensure the reproducibility of the results, SP-STM measurements were carried out on multiple substrates using many tips. The success rate with magnetic tips was $\sim 50 \%$.

STM images obtained using nonmagnetic tips show that the $\mathrm{Mn}_{3} \mathrm{~N}_{2}(010)$ surface consists of a series of identical rows of $\mathrm{Mn}$ atoms. The row heights, however, varied periodically if magnetic tips were used. This row-height modulation was modeled as resulting from the interaction of surface electron spin with the magnetic tip. Therefore, SP-STM images of magnetic materials contain chemical structure and electron spin information. Because the magnetic and nonmagnetic components have different periodicities, they can be separated to yield maps of physical structure and spin behavior. The individual height profiles of both surface maps are in excellent agreement with the simulated ones. Smith is currently applying SP-STM to new magnetic material surfaces, as well as investigating the effects of imaging parameters on magnetic contrast in order to understand spinpolarized tunneling in greater detail.

GREG KHITROV

\section{Nanostructured Copper Achieves Simultaneous High Strength and Ductility}

During the last two decades, there has been a burgeoning of interest in nanostructured metals due to their extremely small grain size and strengths far exceeding those of coarse-grained and even alloyed metals, which can potentially lead to many applications. These materials are generally considered to be nanocrystalline if the average grain or crystallite size is less than $100 \mathrm{~nm}$. However, such materials often exhibit low tensile ductility at room temperature, which limits their utility in shaping operations and load-bearing applications. Therefore, the microstructure may need to be manipulated starting from a uniformly nanocrystalline material. Recently, a group of researchers from the Johns Hopkins University demonstrated that a thermomechanical treatment of $\mathrm{Cu}$ results in a bimodal grain-size distribution with micrometer-sized grains embedded inside a matrix of nanocrystalline and ultrafine grains. With only a moderate population $(\sim 25 \%)$ of the larger grains, such $\mathrm{Cu}$ can achieve a high tensile ductility and, more importantly, 30\% uniform elongation. A key idea to stabilize the large uniform tensile deformation is the efficient use of the larger grains to achieve a strain-hardening rate significantly higher than that predicted for copper in uniaxial tension.

As reported in the October 31 issue of Nature, Y. Wang and colleagues first suppressed dynamic recovery to reach a higher steady-state level of dislocations by rolling the $\mathrm{Cu}$ at liquid nitrogen temperature to a high value of percentage cold work $(\mathrm{CW})$. This led to a lower recrystallization temperature that favored copious nucleation over growth. This allowed the metal to be mostly nanostructured to maintain the high strength of the "composite" material. The larger grains were introduced through secondary recrystallization, that is, abnormal grain growth. The heterogeneous microstructure was found to provide appreciable strain hardening. The proposed mechanisms for enhanced strain hardening include the multiaxial stress states in the inhomogeneous structure, the large strain gradients on the micrometer scale, and deformation twinning. In addition, at the end of the uniform deformation, the larger (softer) grains, which accommodate strains preferentially, have been refined to ultrafine grains that provide large post-uniform elongation. The overall result is an elongation to failure comparable to that of the conventional coarse-grained $\mathrm{Cu}$, but at a strength five to six times higher.

The researchers' thermomechanical approach to the processing of bulk samples improves tensile stability and is also simpler and inexpensive compared with those processes required to produce uniform and truly nanocrystalline grains. Although some of the high strength of the latter may be sacrificed, the new material can reach a better combination of strength and ductility. In addition, the new process avoids the presence of impurities or porosity typical of other processes used to produce nanocrystalline grain.

KINSON C. KAM

\section{Simple Neutron Microscope Uses Refractive Aluminum Lenses}

Neutron imaging is an ideal tool for probing solids and liquids, for the technique complements information gathered from light, x-ray, and electron scattering. One of the drawbacks, thus far, has been the relatively low size and resolution of images obtained through neutron scattering. However, researchers have recently demonstrated a remedy.

In the November 25 issue of Applied Physics Letters, H.R. Beguiristain of Adelphi Technology, California; I.S. Anderson of Institut Laue-Langevin, France; and co-workers report how they used a microscope containing a neutron compound refractive lens (NCRL) to produce high-magnification real images of cadmium, plastic, and steel test objectsanalogous to how a magnifying glass produces images. The scientists constructed the NCRL from 105 individual aluminum biconcave lenses. They designed it to have a focal length $f=0.46 \mathrm{~m}$ for neutrons with a $20-\AA$ wavelength. The researchers placed a multidetector array of $128 \times 128$ pixels at various distances downstream of the NCRL, in order to select a magnification factor and to satisfy the simple lens equation from geometrical optics. Similar to a visible-light microscope, the neutron version has image formation features such as field of view, depth of field, and improved spatial resolution upon magnification. Though the neutron detector pixel size $(7.5 \mathrm{~mm} \times 7.5 \mathrm{~mm})$ and experimental spatial restrictions limited the resolution of the instrument to $214 \mu \mathrm{m}$ at a top magnification of $35 x$, the images are significantly better than simple shadow-graph pictures.

Neutron microscopy extends the reach of traditional imaging techniques, enabling materials to be seen in a new "light." Since neutrons are electrically neutral, they penetrate deeply to the nucleus, and such interactions largely determine their scattering. Furthermore, interactions with the neutron's magnetic 
moment can provide information on magnetic structures, and neutron wavelengths are comparable to atomic length scales. Neutron microscopy will find applications-for example, in morphology and crystallization studies - in probing materials from a variety of scientific and industrial fields.

RICHARD LOUIE

\section{Simulations Indicate Aluminum Has a Higher Ideal Shear Strength than Copper}

The theoretical shear stress or ideal shear stress is the stress necessary to deform a perfect crystal without defects. Usually this value is obtained through theoretical first principles-calculations, although in some instances nanoindentation techniques can serve as an experimental measurement of the ideal shear stress. In the case of aluminum and copper, a recent report of calculations based on density functional theory (DFT) showed that copper has a higher ideal shear strength than aluminum. However, Shigenobu Ogata, Ju Li, and Sidney Yip from the Massachusetts Institute of Technology have found the opposite result also from DFT calculations, as they report in the October 25 issue of Science.

After performing calculations using various DFT methods, results showed that aluminum has the higher ideal shear strength. The scientists followed the same procedure used to obtain the results previously reported, and also used additional methods that further corroborated their results. The researchers based their simulations on a six-atom supercell of three $\{111\}$ layers to calculate the equilibrium lattice constant and the relaxed and unrelaxed $\{111\}<11 \overline{2}>$ shear moduli. A 24-atom supercell of 12 layers for aluminum (10 for copper) served to calculate the intrinsic and unstable stacking-fault energies. The state of stresses considered for the calculations was pure shear or simple shear, with calculated stress values having an uncertainty of $<0.1 \mathrm{GPa}$.

The investigators attribute this result to the fact that the elastic strain of aluminum at the maximum shear stress is more than $50 \%$ larger than that of copper. The ideal pure shear strength of aluminum is $32 \%$ larger than that of copper, despite the fact that the latter has a $25 \%$ larger pure shear modulus along the $\{111\}<11 \overline{2}>$ system. The investigators also attribute this effect to the differences in ion relaxations in both materials: aluminum has almost no relaxation in the $x$ direction, and copper in the $\mathrm{z}$ direction. Therefore, stress applied in the $\langle 110\rangle$ direction hardens copper and softens aluminum, and stress applied in the $\langle 111\rangle$ direction has the opposite effect. As a consequence, interpretation of results from nanoindentation has to account for analysis of the state of stress, said the researchers. Aluminum atoms also have directional bonding which causes a longer shear deformation, higher intrinsic stackingfault energy, and higher ideal shear strength, they said.

SIARI S. SOSA

\section{Neutrons Used for Holographic Imaging of a Pb Crystal}

Electron and x-ray holography are well-known techniques for imaging the atomic structure of condensed materials. However, these methods are still limited because electrons interact strongly with matter, allowing only surface imaging as a practical matter, and $\mathrm{x}$-rays experience great variations of sensitivity for different atomic elements. Recently, L. Cser and Gy. Török from the Central Research Institute for Physics, Hungary, and C. Krexner from the University of Vienna, Austria, proposed experimental setups that enable holography imaging using thermal neutrons with wavelengths close to interatomic distances. The first approach, called an "inside-source concept," is based on large incoherent neutron scattering cross sections of nuclei such as hydrogen that could serve as pointlike sources of neutron spherical waves inside the sample. The second, called an "inside-detector concept," is based on the use of strongly neutronabsorbing isotopes acting as pointlike detectors in the sample.
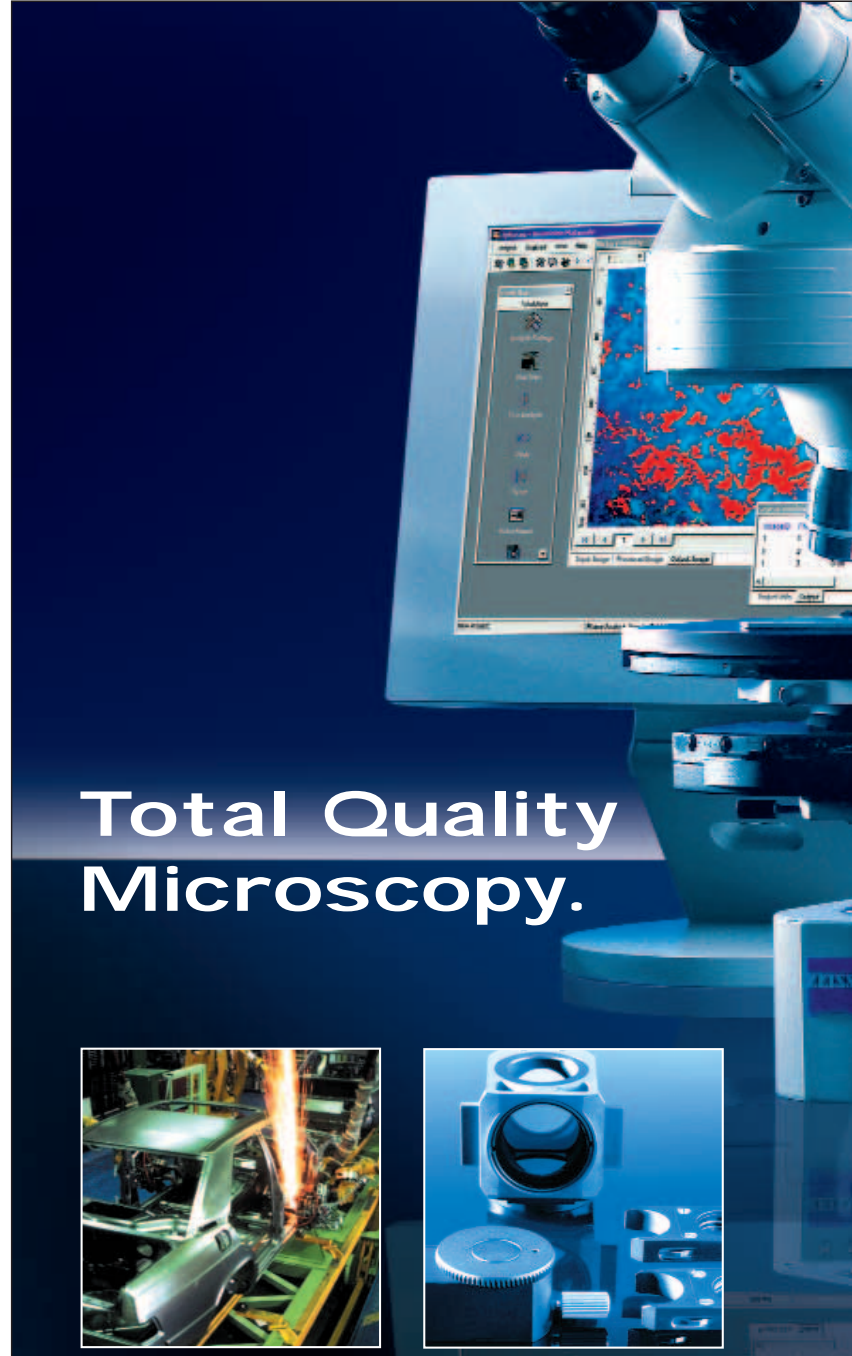

There is a new dynamic force in materials microscopy - advanced materials with increasingly complex properties are demanding new techniques and systems. Now an exciting world of integrated systems for materials microscopy is meeting the challenge of these dynamics - with outstanding materials microscopes, innovative techniques, high resolution digital cameras and superior imaging software. This world of systems is based on the Carl Zeiss philosophy: Total Quality Microscopy.

\section{Materials microscopy on the move.}

Carl Zeiss Microlmaging, Inc. Thornwood, NY 10594 1.800.233.2343 micro@zeiss.com zeiss.com/micro

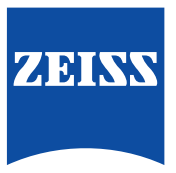

We make it visible. 